\title{
Curves and surfaces in the context of optometry. Part 1: Curves*
}

\section{WF Harris}

Optometric Science Research Group, Department of Optometry, and Department of Mathematics and Statistics, University of Johannesburg, PO Box 524, Auckland Park, 2006 South Africa

<wfh@na.rau.ac.za>

\begin{abstract}
This paper introduces the differential geometry of curves in Euclidean 3-space, the motivation being the writer's belief that, despite their fundamental importance, curves are inadequately treated in optometric educational programs. Curvature and torsion are defined along a curve. Two numerical examples are presented. The fundamental theorem of curves is stated. The relationship of the geometry of varifocal lenses and curves known as involutes are discussed. A brief treatment of the theory of contact is given with suggestions of applications in contact between spectacle lenses and frames, contact lenses and corneas (including orthokeratology), intra-ocular lenses and structures in the eye, and spectacle frames and the face.
\end{abstract}

Keywords: Curves, curvature, torsion, varifocal lenses, theory of contact, differential geometry

Despite their fundamental importance in optometry curves and surfaces are treated superficially and naïvely in optometric educational programs. This paper (on curves) and a second $^{1}$ (on surfaces) attempt to outline a proper approach to curves and surfaces in the context of optometry. The purpose is to introduce the mathematical discipline known as differential geometry and to point to some optometric applications. The intention, however, is not to cover the material in detail; there are good texts ${ }^{2,3}$ that do that. The intention is rather to attempt to present something of the character of differential geometry. It is the author's hope that the reader will gain enough insight to be able to follow the mechanics of the mathematics in the examples in the Appendix.

We review the concept of the vector in Euclidean 3-space, vector functions of a scalar variable, derivatives of the functions and parametric representations. A curve is defined as a set of parametrizations. Curvature and torsion are defined along a curve. The fundamental theorem of curves in space deals with the existence and uniqueness of curves of given curvature and torsion. Finally involutes and the theory of contact are discussed. The former have a bearing on the geometry of varifocal lenses and the latter on several matters of optometric importance including contact between spectacle lens and frame and contact lens and cornea and orthokeratology.

This paper draws heavily on the text by Lipschutz .
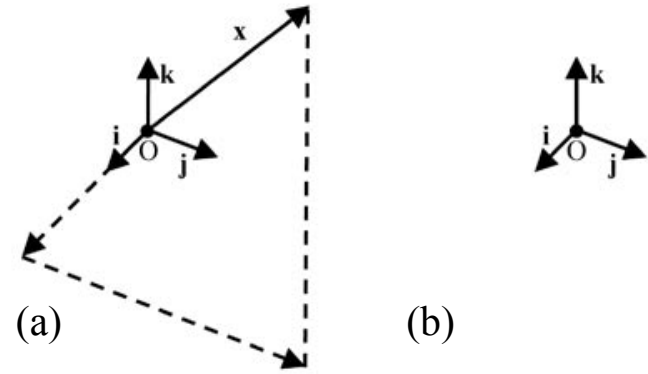

Figure 1. Representation of a vector $\mathbf{x}$ in Euclidean 3-space. $\mathrm{O}$ is the origin. $\mathbf{i}, \mathbf{j}$ and $\mathbf{k}$ are three mutually perpendicular unit vectors. $\mathbf{x}=3 \mathbf{i}+4 \mathbf{j}+5 \mathbf{k}$. $\mathbf{x}$ is represented by an arrow in (a) and a dot in (b). 


\section{Vectors in Euclidean 3-space}

Figure 1 is a graphical representation of a three-dimensional Euclidean space. There is an origin $\mathrm{O}$ and three mutually-orthogonal unit vectors $\mathbf{i}, \mathbf{j}$ and $\mathbf{k}$. $\mathbf{x}$ is a vector in the space. It can be expressed as a linear combination of the unit vectors as

$\mathbf{x}=3 \mathbf{i}+4 \mathbf{j}+5 \mathbf{k}$

or we can write it as the coordinate vector

$\mathbf{x}=\left(\begin{array}{l}3 \\ 4 \\ 5\end{array}\right)$

Vector $\mathbf{x}$ has magnitude or length given by the generalization of Pythagoras's equation

$x=|\mathbf{x}|=\sqrt{3^{2}+4^{2}+5^{2}}$.

In general we can write

$\mathbf{x}=\left(\begin{array}{l}x_{1} \\ x_{2} \\ x_{3}\end{array}\right)$

and

$x=|\mathbf{x}|=\sqrt{x_{1}^{2}+x_{2}^{2}+x_{3}^{2}}$.

If one is working entirely in a plane then two coordinates are usually sufficient and equations 1 and 2 can be reduced to

$\mathbf{x}=\left(\begin{array}{l}x_{1} \\ x_{2}\end{array}\right)$

and

$x=|\mathbf{x}|=\sqrt{x_{1}^{2}+x_{2}^{2}}$.

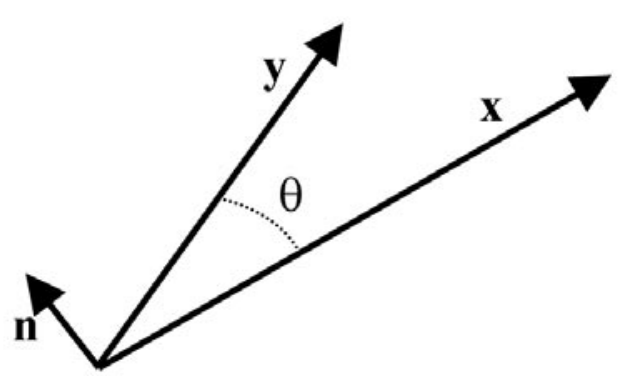

Figure 2. Two vectors $\mathbf{x}$ and $\mathbf{y}$ in Euclidean space with angle $\theta$ between them. The scalar product is defined by equation 5 and the vector product by equation $6 . \mathbf{n}$ is a unit vector orthogonal to the plane containing $\mathbf{x}$ and $\mathbf{y}$.
Two vectors $\mathbf{x}$ and $\mathbf{y}$ (Figure 2) that make an angle $\theta$ with one another can be multiplied in two important ways. We represent them as

$\mathbf{x} \bullet \mathbf{y}=x y \cos \theta$

and

$\mathbf{x} \times \mathbf{y}=\mathbf{n} x y \sin \theta$.

$\mathbf{n}$ is one of the two unit vectors orthogonal to the plane containing $\mathbf{x}$ and $\mathbf{y}$. Of the two possibilities $\mathbf{n}$ is the one with direction in which a right-handed screw would advance if turned so that $\mathbf{x}$ was rotated through $\theta$ towards $\mathbf{y}$. The first product (equation 5) is often called the dot, scalar or inner product and the second (equation 6) as the cross or vector product of $\mathbf{x}$ and $\mathbf{y}$. Although perhaps not immediately obvious the dot product is equivalent to the product often written $\mathbf{x}^{\prime} \mathbf{y}$ in linear algebra, the prime representing the matrix transpose.

\section{Vector Function of a Scalar Variable}

Let $\mathbf{x}$ be a function of a scalar $t$ defined on an interval $I$ of the real number line. We write it as

$\mathbf{x}=\mathbf{f}(t) \quad t \in I$.

It will be convenient to write equation 7 as

$\mathbf{x}=\mathbf{x}(t) \quad t \in I$.

(Strictly-speaking equation 8 is an abuse of mathematical notation because the symbol $\mathbf{x}$ means two different things at the same time: a function on the right-hand side of the equality and the value of the function on the left-hand side. The context, however, will always make the meaning clear in any given case.) Equation 8 can be thought of as containing three scalar functions of the variable $t$ :

$\mathbf{x}=\left(\begin{array}{l}x_{1}(t) \\ x_{2}(t) \\ x_{3}(t)\end{array}\right) \quad t \in I$.

For each value of the parameter $t$ in the interval $I$ there is a corresponding vector $\mathbf{x}$. Together all the vectors $\mathbf{x}$ form a curve in space as in Figure 3. In effect equation 8 is a rule for distorting interval $I$; the straight line segment becomes the curve. As $t$ increases from $a$ in the interval $I=[a, b]$ vector $\mathbf{x}$ changes from $\mathbf{x}(a)$ at one end of the curve finally reaching the 


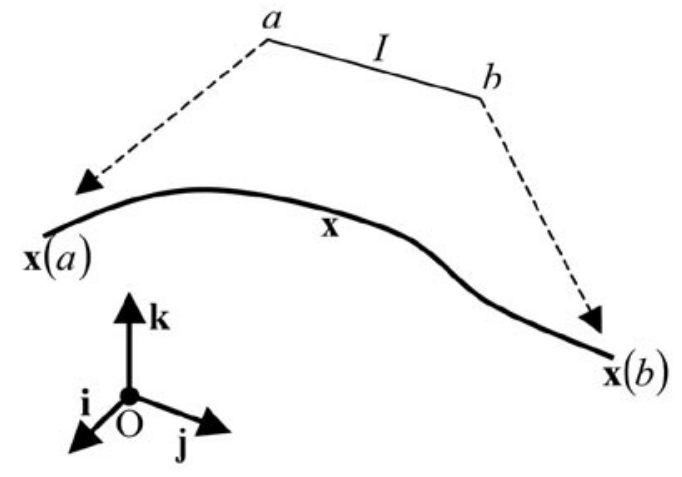

Figure 3. A vector function $\mathbf{x}=\mathbf{x}(t)$ defined on the interval $t \in I$ and represented in Euclidean space.

other end at $\mathbf{x}(b)$ when $t=b$. One can think of the curve as starting at $t=a$ and ending at $t=$ $b$ in which case increasing $t$ defines a direction along the curve; the curve is then said to be an oriented curve.

The following is an example of a vector function of a scalar variable:

$$
\mathbf{x}=\left(\begin{array}{c}
(2 \cos \theta-1) \cos \theta \\
(2 \cos \theta-1) \sin \theta
\end{array}\right) \quad \theta \in[0,2 \pi]
$$

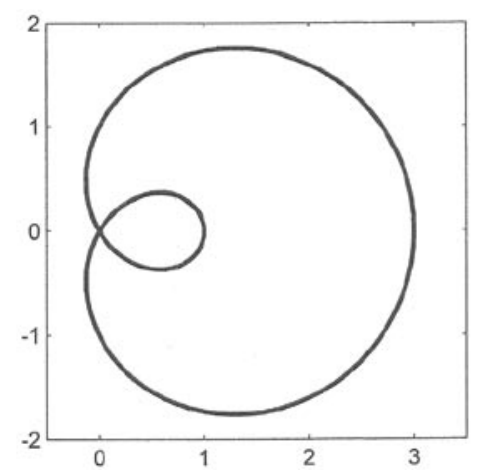

Figure 4. Graphical representation of the parametrization defined by equation 10 .

With only two components its graphical representation is planar (Figure 4). Traversing the interval from 0 to $2 \pi$ we see that $\mathbf{x}$ begins at $\mathbf{x}(0)=\left(\begin{array}{l}1 \\ 0\end{array}\right)$ and traverses the curve in an anticlockwise sense arriving finally at $\mathbf{x}(2 \pi)$ which is where it started. The curve is closed. Notice also that the curve intersects itself at the point $\left(\begin{array}{l}0 \\ 0\end{array}\right)$, that is, where $\theta=\pi / 3$ and $5 \pi / 3$. One can show that the same curve can be represented by the equation

$x^{4}-4 x^{3}+3 x^{2}+2 x^{2} y^{2}-4 x y^{2}-y^{2}+y^{4}=0$ in terms of Cartesian coordinates $x$ and $y$.

\section{Derivatives}

For some particular value, say $t_{0}$, of the parameter $t$ in the interval $I$ the derivative of the function $\mathbf{x}=\mathbf{x}(t)$ is defined by means of

$\frac{d \mathbf{x}}{d t}\left(t_{0}\right)=\lim _{t \rightarrow t_{0}} \frac{\mathbf{x}(t)-\mathbf{x}\left(t_{0}\right)}{t-t_{0}}$

provided the limit exists. Commonly one abbreviates the derivative $\frac{d \mathbf{x}}{d t}\left(t_{0}\right)$ as $\mathbf{x}^{\prime}\left(t_{0}\right)$. As is evident from Figure 5 the derivative $\mathbf{x}^{\prime}(t)$ is a vector tangent to the curve at the value $t$ of the parameter.
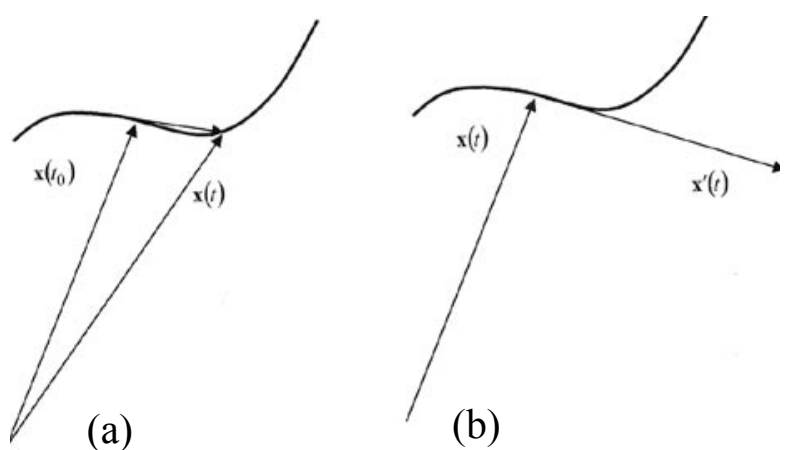

(b)

Figure 5. The tangent vector $\mathbf{x}^{\prime}(\mathrm{t})$ at the point with parameter $t$. It is defined by equation 11 .

Higher-order derivatives may also be defined. The second and third derivatives are written $\mathbf{x}^{\prime \prime}(t)$ and $\mathbf{x}^{\prime \prime \prime}(t)$ respectively. The $p$ th derivative is written $\mathbf{x}^{(p)}(t)$.

Derivatives are used to define what is called the class of a function. We define the class of a function here and use it below but the reader may find it helpful to skip over all references to the concept on first reading. A function $\mathbf{x}=\mathbf{x}(t)$ $t \in I$ is said to be of class $C^{m}$ if $\mathbf{x}^{(m)}(t)$ exists and is continuous on the interval $I$. Notice that a function of class $C^{m}$ is also of class $C^{m-1}$. Derivatives of all orders exist for the function defined by equation 10; the function is said to have class $C^{\infty}$.

\section{Parametrizations}

It can happen that the first derivative of a function is null at some point along the curve. The curve in that case has no tangent at the 
point. We avoid difficulties that then arise in the mathematics by eliminating such functions from further consideration. The functions that remain are what we call regular parametrizations; they are functions with $\mathbf{x}^{\prime}(t) \neq \mathbf{0}$ everywhere. More formally a regular parametrization is a function $\mathbf{x}=\mathbf{x}(t) \quad t \in I$ such that $\mathbf{x}(t)$ is of class $C^{1}$ in $I$ and $\mathbf{x}^{\prime}(t) \neq \mathbf{o}$ for all $t$ in $I$.

Equation 10 is an example of a regular parametrization. Here is another

$\mathbf{x}=\left(\begin{array}{l}\left(2 \cos t^{2}-1\right) \cos t^{2} \\ \left(2 \cos t^{2}-1\right) \sin t^{2}\end{array}\right) \quad t \in[0, \sqrt{2 \pi}]$.

(a)

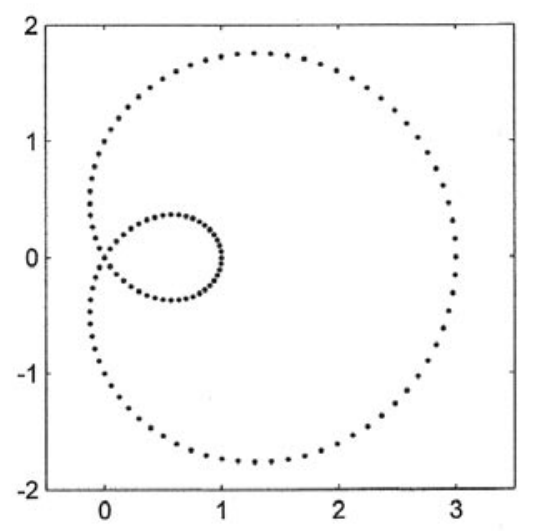

(b)

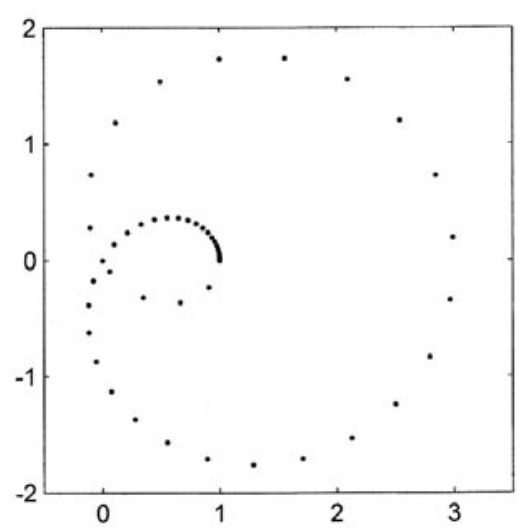

Figure 6. Equation 10 plotted for $\theta=i \pi / 60$ in (a) and equation 12 plotted for $t=i \sqrt{\pi / 1200}$ in (b). $i$ is an integer such that the parameters $\theta$ and $t$ lie on the intervals $I$.

The two parametrizations are different although they do seem to have similar features. Despite the fact that they are different the two parametrizations have exactly the same geometrical representation, namely Figure 4. Differences are only in the way the curve is drawn. Figure 6 shows the functions plotted for particular values of the parameters: (a) is obtained from equation 10 with those values of $\theta$ in $[0,2 \pi]$ that are multiples of $\pi / 60$ and (b) from equation 12 with values of $t$ in $[0, \sqrt{2 \pi}]$ that are multiples of $\sqrt{\pi / 1200}$.

\section{Curves}

Informally what is illustrated in Figure 4 one may call a curve. However it is really no more than a geometrical representation of a curve. That it is an imperfect representation is apparent from the fact that it has a nonzero thickness, for example, which the true curve does not have. No drawing can fully and precisely capture the true nature of a curve. Formally a curve is an abstraction not necessarily related to any drawing although one can frequently illustrate many of the features of a curve by means of a drawing.

As we have seen two different parametrizations can generate the same geometrical representation. One can in fact find any number that will do the same thing. More formally a curve is the set of all the parametrizations that generate the same geometrical representation. Our concern is only with regular parametrizations and, hence, only with regular curves.

Consider a regular curve generated by $\mathbf{x}=$ $\mathbf{x}(t) \quad t \in I$. If $t_{1} \neq t_{2}$ implies that $\mathbf{x}\left(t_{1}\right) \neq \mathbf{x}\left(t_{2}\right)$ then the curve is called simple. The curve represented in Figure 4 is not simple.

From point with parameter $t_{1}$ to point with parameter $t_{2}\left(t_{1} \leq t_{2}\right)$ a regular curve has length given by

$$
s=\int_{t_{1}}^{t_{2}}\left|\mathbf{x}^{\prime}(t)\right| d t
$$

\section{Natural Parameter}

Arc length $s$ along a curve can be used as a parameter. It is then referred to as a natural parameter. The parametrization then is $\mathbf{x}=\mathbf{x}(s)$ $s \in I$. Differentiation with respect to a natural parameter is usually represented by means of a dot instead of a prime. Thus $\dot{\mathbf{x}}(s)=\frac{d \mathbf{x}}{d s}(s)$, $\ddot{\mathbf{x}}=\frac{d^{2} \mathbf{x}}{d s^{2}}(s)$, etc. Like $\mathbf{x}^{\prime}(t) \dot{\mathbf{x}}(s)$ is a vector tangent to the curve. More particularly $\dot{\mathbf{x}}(s)$ is a unit vector. One writes the unit tangent vector as

$\mathbf{t}=\dot{\mathbf{x}}$. 
(a)

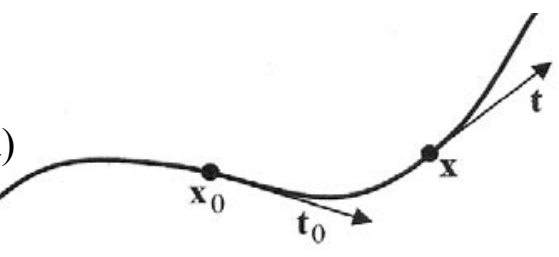

(b)

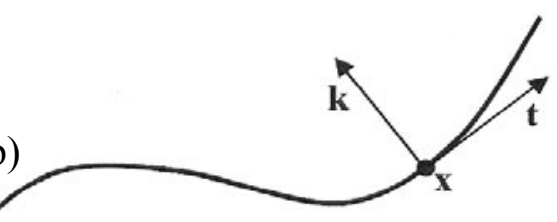

Figure 7. The unit tangent vector $\mathbf{t}$ (defined by equation 14) changes along a curve. The curvature vector $\mathbf{k}$ (defined by equation 15) represents the nature of that change.

\section{Curvature}

Along a straight line the unit tangent vector is constant and, hence, $\ddot{\mathbf{x}}=\mathbf{o}$. In general $\ddot{\mathbf{x}}$ is a measure of how the curve bends. It is called the curvature vector and written

$\mathbf{k}=\ddot{\mathbf{x}}$

See Figure 7. The magnitude of the curvature vector is the curvature $\kappa$, that is

$\kappa=|\mathbf{k}|$.

The unit vector

$\mathbf{n}=\frac{\mathbf{k}}{\kappa}$

is called the principal unit normal to the curve (Figure 8).

The unit binormal is defined by

$\mathbf{b}=\mathbf{t} \times \mathbf{n}$.

$\mathbf{t}, \mathbf{n}$ and $\mathbf{b}$ are a triplet of mutually orthogonal unit vectors. They in turn define the osculating

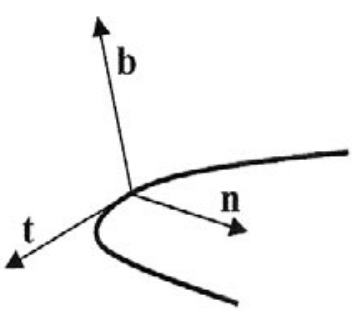

(a)

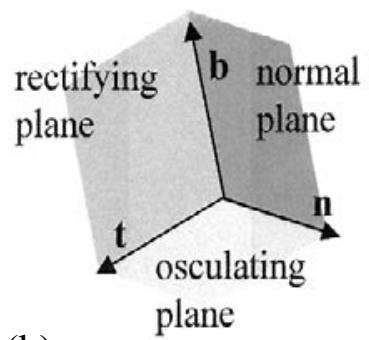

(b)
Figure 8. The unit tangent vector $\mathbf{t}$, the principal unit normal $\mathbf{n}$, the unit binormal $\mathbf{b}$ and three planes they define at a point on a curve in space. plane, the normal plane and the rectifying plane (Figure 8) for the curve at a point on it.

\section{Torsion}

In the case of a curve that lies in a plane the unit binormal $\mathbf{b}$ is fixed $(\dot{\mathbf{b}}=\mathbf{0})$ and is normal to the plane. In general a space curve 'twists' out of the plane according to the vector $\dot{\mathbf{b}}$. The curve has a torsion $\tau$ defined by

$\dot{\mathbf{b}}=-\tau \mathbf{n}$.

\section{From Curve to Curvature and Torsion}

From the definitions it turns out that the curvature and torsion of a curve are given by

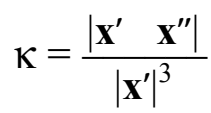

and

$\tau=\frac{\left|\mathbf{x}^{\prime} \cdot \mathbf{x}^{\prime \prime} \times \mathbf{x}^{\prime \prime \prime}\right|}{\left|\mathbf{x}^{\prime} \times \mathbf{x}^{\prime \prime}\right|^{2}}$

respectively.

From these equations it is evident that calculation of curvature and torsion at any point on a given curve is routine. See the Appendix for examples. The reverse problem of finding a curve with given curvature and torsion properties along it is more of a challenge.

\section{From Curvature and Torsion to Curve}

Suppose we have two continuous functions $\kappa(s)$ and $\tau(s)$ defined on an interval $[a, b]$. Then the fundamental theorem of curves in space tells us that there exists one and only one space curve for which $\kappa(s)$ is the curvature, $\tau(s)$ the torsion and $s$ a natural parameter. (For this purpose two curves are regarded as the same if they differ only in position and orientation in space.)

The fundamental theorem guarantees existence and uniqueness of the curve, but finding the curve is another matter. The equation of the curve is the vector function $\mathbf{x}(s)$ that satisfies the set of equations

$\dot{\mathbf{t}}=\kappa \mathbf{n}$

$\dot{\mathbf{n}}=-\kappa \mathbf{t}+\tau \mathbf{b}$

$\dot{\mathbf{b}}=-\tau \mathbf{n}$. 
These are called the Serret-Frenet equations of a curve. Equation 22 comes from equations 14, 15 and 17; equation 24 is equation 19; and equation 23 follows from differentiating $\mathbf{n}=\mathbf{b} \times \mathbf{t}$. The reader interested in the problem of solving for the curve is referred elsewhere ${ }^{2,3}$.

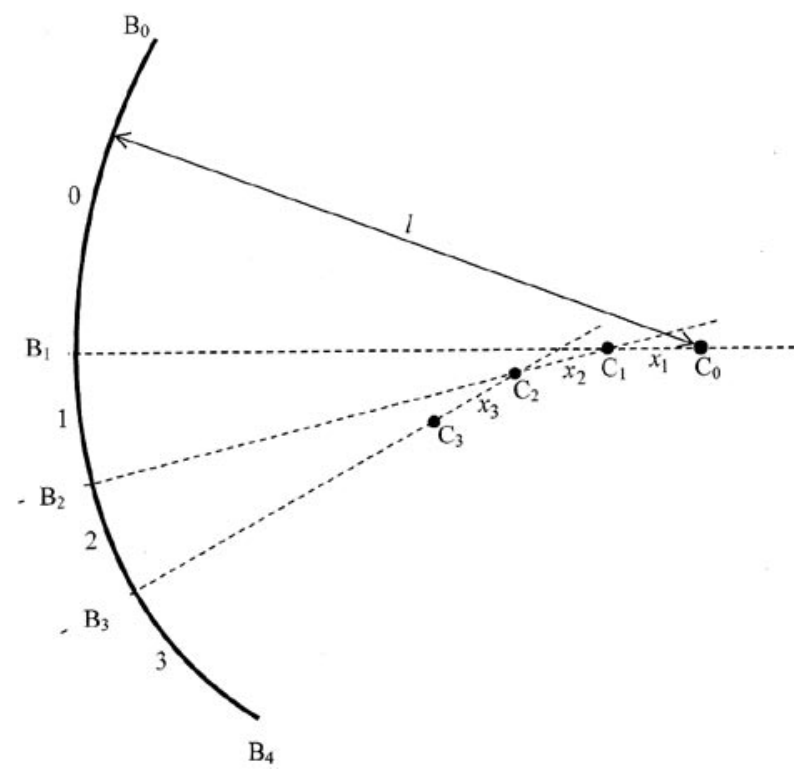

Figure 9. Cross-section of the front surface $\mathrm{B}_{0} \mathrm{~B}_{4}$ of a 4-focal lens. Arcs $0,1,2$ and 3 have centres of curvature at $\mathrm{C}_{0}, \mathrm{C}_{1}$, $\mathrm{C}_{2}$ and $\mathrm{C}_{3}$. Arc 0 represents the distance portion, arcs 1 and 2 , intermediate portions and arc 3 the near portion. Arc 0 has radius of curvature $l$, arc 1 has radius of curvature $l-x_{1}$, arc 2 has $l-x_{1}-x_{2}$ and arc 3 has $l-x_{1}-x_{2}-x_{3}$. Instead of discrete points $\mathrm{C}_{0}, \mathrm{C}_{1}, \mathrm{C}_{2}$ and $\mathrm{C}_{3}$ a varifocal has a curve $\mathrm{C}$. The crosssection of the front surface of the lens is an involute $\mathrm{B}$ of $\mathrm{C}$.

\section{Multifocals and Varifocals}

Associated with a curve are other curves called involutes. They have a bearing on multifocals and varifocals.

Imagine a board into which pins are stuck as shown in Figure 9 at points $\mathrm{C}_{0}, \mathrm{C}_{1}, \mathrm{C}_{2}$ and $\mathrm{C}_{3}$. One end of a piece of string of length $l$ is attached to the pin at $\mathrm{C}_{0}$. A drawing is now constructed with a pencil attached at the other end while the string is kept taught. Starting at $\mathrm{B}_{0}$ the pencil draws an arc of radius $l$ and centre $C_{0}$. When the pencil reaches $\mathrm{B}_{1}$ the string encounters the pin at $C_{1}$. Between $B_{1}$ and $B_{2}$ the arc has centre at $\mathrm{C}_{1}$ and radius $l-x_{1}$. Between $\mathrm{B}_{2}$ and $\mathrm{B}_{3}$ the arc has centre $\mathrm{C}_{2}$ and radius $l-x_{1}-x_{2}$. Finally between $\mathrm{B}_{3}$ and $\mathrm{B}_{4}$ the centre is $\mathrm{C}_{3}$ and the radius is $l-x_{1}-x_{2}-x_{3}$. One can think of the arcs as the vertical section of the front surface of a multifocal lens, a 4-focal in this case. Let the lens be in air and have index of refraction $n$. Then the distant portion (arc 0) has power $(n-1) / l$, the first intermediate portion (arc 1) has power $\frac{n-1}{l-x_{1}}$, the second intermediate portion $(\operatorname{arc} 2) \frac{n-1}{l-x_{1}-x_{2}}$ and the reading portion $(\operatorname{arc} 3)$ $\frac{n-1}{l-x_{1}-x_{2}-x_{3}}$.

By inserting more pins on an arc between $\mathrm{C}_{0}$ and $\mathrm{C}_{3}$ one can construct the cross section of an $n$-focal where $n$ is some higher integer. The more pins there are the closer do they approximate a smooth curve $\mathrm{C}$ and the closer the section $\mathrm{B}$ approaches that of a varifocal. The relevance of this here is that $B$ is an example of what, in differential geometry, is called an involute of curve C. C is an evolute of B. In general terms an involute is a curve traced by the end of taught string unwound from (or wound onto) a space curve.

\section{Contact}

The next topic commonly encountered in a course on differential geometry is what is called the theory of contact. It has considerable applications in engineering and, indeed, considerable potential applications in optometry.

The equation of a surface can be written as

$F(\mathbf{x})=0$

where $F(\mathbf{x})$ is a function of the vector $\mathbf{x}$. As an example consider the surface of a sphere of radius $a$. We could write its equation as

$x_{1}^{2}+x_{2}^{2}+x_{3}^{2}=a^{2}$

or as

$x_{1}^{2}+x_{2}^{2}+x_{3}^{2}-a^{2}=0$.

The latter is of the same form as equation 25.

Consider a surface (equation $F(\mathbf{x})=0$ ) and a curve (equation $\mathbf{x}=\mathbf{x}(t)$ ) in space. They may or may not intersect or touch each other. We define the function $f(t)=F(\mathbf{x}(t))$. 
If $f(t) \neq 0$ then the surface and the curve are not in contact at the point on the curve with parameter $t$. If $f(t)=0$ then they are in contact there. Degrees of contact are defined. If

$f(t)=f^{\prime}(t)=f^{\prime \prime}(t)=\ldots=f^{(n-1)}(t)=0$

but

$f^{(n)}(t) \neq 0$

then there is $n$-degree contact at the point.

The higher the degree of contact the more snug or intimate is the contact at the point. One would expect less stress and deformation and greater mechanical stability and thermal conduction at points of higher degree of contact. One would also expect better charge transfer with electrical contacts of higher degree.

Optometric applications immediately spring to mind: contact between lens and frame and the problem of chipping and cracking; contact between the frame and the face; contact between the contact lens and the cornea; orthokeratology; contact between the haptic of an intra-ocular lens and structures in the eye and the problem of the positional stability of the lens.

\section{Concluding Remarks}

We have given here a brief outline of the differential geometry of curves. For more detail the reader could consult standard references ${ }^{2,3}$.

Mention has been made of application in varifocal lenses and to problems of contact between lenses and frames and lenses and anatomical structures. No doubt there are many more possibilities.

A subsequent paper $^{1}$ introduces the differential geometry of surfaces.

\section{Acknowledgement}

This paper is based on research supported under Grant Number 2053699 to WF Harris by the National Research Foundation.

\section{References}

1. Harris WF. Curves and surfaces in the context of optometry. Part 2: Surfaces. S Afr Optom 200665 submitted herewith.
2. Struik DJ. Lectures on Classical Differential Geometry. Addison-Wesley, Reading, MA, 1950.

3. Lipschutz, MM. Schaum's Outline of Theory and Problems of Differential Geometry. McGraw-Hill, New York, 1969.

\section{Appendix}

\section{Example 1}

We calculate the curvature at points along the curve in Figure 4. We make use of the parametrization given by equation 10. Differentiation gives

$\mathbf{x}^{\prime}=\left(\begin{array}{c}-2 \sin 2 \theta+\sin \theta \\ 2 \cos 2 \theta-\cos \theta\end{array}\right)$

and

$\mathbf{x}^{\prime \prime}=\left(\begin{array}{l}-4 \cos 2 \theta+\cos \theta \\ -4 \sin 2 \theta+\sin \theta\end{array}\right)$

Hence

$\mathbf{x}^{\prime} \times \mathbf{x}^{\prime \prime}=\left|\begin{array}{ccc}-2 \sin 2 \theta+\sin \theta & -4 \cos 2 \theta+\cos \theta & \mathbf{i} \\ 2 \cos 2 \theta-\cos \theta & -4 \sin 2 \theta+\sin \theta & \mathbf{j} \\ 0 & 0 & \mathbf{k}\end{array}\right|$

which simplifies to

$\mathbf{x}^{\prime} \times \mathbf{x}^{\prime \prime}=(9-6 \cos \theta) \mathbf{k}$.

Also

$\left|\mathbf{x}^{\prime}\right|^{2}=5-4 \cos \theta$.

Hence, from Equation 20, the curvature is

$\kappa=\frac{9-6 \cos \theta}{(5-4 \cos \theta)^{3 / 2}}$.

As expected the curvature is a function of the parameter $\theta$. Its maximum, 3 units, occurs at $\theta=$ 0 . It is $2 / \sqrt{3}$ units at $\theta=\pi / 3$ (the self-intersection) and a minimum of $5 / 9$ units at $\theta=\pi$.

\section{Example 2}

We calculate the curvature and torsion along a helix. We begin with the parametrization

$\mathbf{x}=\left(\begin{array}{c}a \cos t \\ a \sin t \\ b t\end{array}\right)$. 
The derivatives are

$$
\begin{aligned}
& \mathbf{x}^{\prime}=\left(\begin{array}{c}
-a \sin t \\
a \cos t \\
b
\end{array}\right), \\
& \mathbf{x}^{\prime \prime}=\left(\begin{array}{c}
-a \cos t \\
-a \sin t \\
0
\end{array}\right)
\end{aligned}
$$

Both curvature and torsion in this case are independent of the parameter. They are the same everywhere on the helix.

and

$\mathbf{x}^{\prime \prime \prime}=\left(\begin{array}{c}a \sin t \\ -a \cos t \\ 0\end{array}\right)$.

Hence

$\mathbf{x}^{\prime} \times \mathbf{x}^{\prime \prime}=\left|\begin{array}{ccc}-a \sin t & -a \cos t & \mathbf{i} \\ a \cos t & -a \sin t & \mathbf{j} \\ b & 0 & \mathbf{k}\end{array}\right|$

or

$\mathbf{x}^{\prime} \times \mathbf{x}^{\prime \prime}=\left(\begin{array}{c}a b \sin t \\ -a b \cos t \\ a^{2}\end{array}\right)$.

Hence

$\left|\mathbf{x}^{\prime} \times \mathbf{x}^{\prime \prime}\right|^{2}=a^{2}\left(a^{2}+b^{2}\right)$

and

$\left|\mathbf{x}^{\prime}\right|^{2}=a^{2}+b^{2}$.

Also

$\mathbf{x}^{\prime} \cdot \mathbf{x}^{\prime \prime} \mathbf{x} \mathbf{x}^{\prime \prime \prime}=\operatorname{det}\left(\begin{array}{ccc}-a \sin t & -a \cos t & a \sin t \\ a \cos t & -a \sin t & -a \cos t \\ b & 0 & 0\end{array}\right)=a^{2} b$.

Equations 20 and 21 then give the curvature

$\kappa=\frac{\sqrt{a^{2}}}{a^{2}+b^{2}}$

and the torsion

$\tau=\frac{b}{a^{2}+b^{2}}$. 